\title{
Knowledge, attitude, and practice of artificial (i) intelligence in emergency and trauma surgery, the ARIES project: an international web-based
} survey

Belinda De Simone ${ }^{1 *}$, Fikri M. Abu-Zidan², Andrew A. Gumbs', Elie Chouillard', Salomone Di Saverio ${ }^{3}$, Massimo Sartelli ${ }^{4}$, Federico Coccolini ${ }^{5}$, Luca Ansaloni ${ }^{6}$, Toby Collins ${ }^{7}$, Yoram Kluger $^{8}$, Ernest E. Moore ${ }^{9}$, Andrej Litvin ${ }^{10}$, Ari Leppaniemi ${ }^{11}$, Pietro Mascagni ${ }^{12}$, Luca Milone ${ }^{13}$, Micaela Piccoli $^{14}$, Mohamed Abu-Hilal ${ }^{15}$, Michael Sugrue ${ }^{16}$, Walter L. Biffl ${ }^{17}$ and Fausto Catena ${ }^{18}$

\begin{abstract}
Aim: We aimed to evaluate the knowledge, attitude, and practices in the application of Al in the emergency setting among international acute care and emergency surgeons.

Methods: An online questionnaire composed of 30 multiple choice and open-ended questions was sent to the members of the World Society of Emergency Surgery between 29th May and 28th August 2021. The questionnaire was developed by a panel of 11 international experts and approved by the WSES steering committee.

Results: 200 participants answered the survey, 32 were females (16\%). 172 (86\%) surgeons thought that Al will improve acute care surgery. Fifty surgeons (25\%) were trained, robotic surgeons and can perform it. Only 19 (9.5\%) were currently performing it. 126 (63\%) surgeons do not have a robotic system in their institution, and for those who have it, it was mainly used for elective surgery. Only 100 surgeons (50\%) were able to define different Al terminology. Participants thought that $\mathrm{Al}$ is useful to support training and education (61.5\%), perioperative decision making (59.5\%), and surgical vision (53\%) in emergency surgery. There was no statistically significant difference between males and females in ability, interest in training or expectations of Al ( $p$ values $0.91,0.82$, and 0.28 , respectively, MannWhitney $\mathrm{U}$ test). Ability was significantly correlated with interest and expectations ( $p<0.0001$ Pearson rank correlation, rho 0.42 and 0.47 , respectively) but not with experience $(p=0.9$, rho -0.01$)$.
\end{abstract}

Conclusions: The implementation of artificial intelligence in the emergency and trauma setting is still in an early phase. The support of emergency and trauma surgeons is essential for the progress of Al in their setting which can be augmented by proper research and training programs in this area.

Keywords: Artificial intelligence, Emergency surgery, Trauma surgery, Research, Survey, Decision making, Robotic surgery, Laparoscopy

\footnotetext{
*Correspondence: desimone.belinda@gmail.com

${ }^{1}$ Department of Emergency and Metabolic Minimally Invasive Surgery,

Centre Hospitalier Intercommunal de Poissy/Saint Germain en Laye, 10 Rue de Champ Gaillard, Poissy Cedex, France

Full list of author information is available at the end of the article
}

\section{Background}

Artificial Intelligence (AI) is defined as the study of algorithms that give machines the ability to perform humanlike tasks and perform cognitive functions that they were not necessarily programmed for such as problem-solving, original author(s) and the source, provide a link to the Creative Commons licence, and indicate if changes were made. The images or other third party material in this article are included in the article's Creative Commons licence, unless indicated otherwise in a credit line to the material. If material is not included in the article's Creative Commons licence and your intended use is not permitted by statutory regulation or exceeds the permitted use, you will need to obtain permission directly from the copyright holder. To view a copy of this licence, visit http://creativecommons.org/licenses/by/4.0/. The Creative Commons Public Domain Dedication waiver (http://creativeco mmons.org/publicdomain/zero/1.0/) applies to the data made available in this article, unless otherwise stated in a credit line to the data. 
object and word recognition, and decision-making [1]. It is a very complex branch of computer engineering that covers various fields of research including machine learning, natural language processing, artificial neural networks, and computer vision [main definitions are summarised in Additional file 1: Appendix 1]. It can be applied in the daily practice of medicine and has had an exponential increase in interest over the last few years. It is gradually supporting surgical practice using technological advancements in imaging, patient management navigation, and robotic interventions [2]. Surgery is associated with AI in robotic surgery which is a novel evolution of minimally invasive surgery aiming to improve surgical outcomes and patient's experience [3].

To our knowledge, there are few articles focused on the utility of AI technologies in emergency and trauma surgery specifically [4-7]. Emergency surgeons take decisions that have major impacts on patients. This needs an accurate assessment of the patient's clinical and radiological data to have a favourable clinical outcome. Accordingly, AI can be very useful in these highly demanding, stressful, and serious situations. The Artificial Intelligence in Emergency and Trauma Surgery (ARIES) survey aimed to evaluate the knowledge, attitude, and practices in the application of AI in the emergency setting among international acute care and emergency surgeons.

\section{Methods}

\section{Ethical considerations}

This survey evaluated the perception, attitude, and knowledge of emergency and trauma surgeons on the use and application of AI in emergency and trauma surgery. Participation in the survey was voluntary, and the participants' data were anonymized. No personally identifiable data were collected. Accordingly, ethical approval was not required.

\section{Study design}

A cross-sectional study among the members of the World Society of Emergency Surgery (WSES) between 29th May and 28th August 2021 (3 months period).

\section{Sample size}

We have sent the questionnaire to 10,000 mailing list's members of the World Society of Emergency Surgery (WSES). Accordingly, sample size calculation was not needed. The response rate was $2 \%(200 / 10000)$.

\section{Questionnaire design}

The main objectives of the survey were to (1) assess the interest of acute care surgeons in AI application in the management of acute care surgical patients and to (2) define priorities of research projects in the implementation of AI in trauma and acute care surgery.

This online questionnaire was composed of 30 multiple choice and open questions (Additional file 1: Appendix 1) and was designed following the Checklist for Reporting Results of Internet E-Surveys (CHERRIES) [8-11]. The questionnaire was divided into 5 sections: (1) Demographic: questions (1-8), (2) Skill and technology questions (9-16), (3) AI knowledge questions (17-19), (4) Expectations and involvement questions (20-27), and (5) Suggestions questions (28 to 30 ).

The questionnaire was initially written by BDS. It was then sent to an international expert panel consisting of 10 members for their advice and modifications including members of the Editorial Board of the Artificial Intelligence Surgery Journal, especially of PM and TC, authors of a previous survey about AI [https://forms.gle/hHeyE v3EE3Ff3Vcm7], and AG who gave guidance on the questions regarding autonomous actions. Finally, the definitive questionnaire was submitted to the WSES steering committee to be endorsed.

\section{Validity and piloting}

We depended mainly on surface validity for validation while content validity depended mainly on the experts' experience in this area. We did not pilot the questionnaire for linguistic clarity because it was reviewed by 9 international experts who stemmed from 5 different countries speaking different mother tongue languages including English, Arabic, French, Italian, and Russian assuring the clarity of the questionnaire for the international participants.

\section{Distribution of the survey and data collection}

The online survey was built upon a google form platform which was accessed through the WSES website. The survey was sent by mail to the WSES members through the mailing list. Data were collected and stored in an online database protected by a password known only by the principal investigator. The survey was announced, advertised, and diffused by the WSES website with a programmed invitation to participate sent by mail, for 3 months (29th May to 28th August 2021).

\section{Statistical analysis}

Statistical analysis was carried out using non-parametric methods. Mann Whitney U test was used to compare two independent groups. Kruskal-Wallis test was used to compare three independent groups while Pearson rank correlation was used to correlate different ranks. A $\mathrm{p}$-value of less than 0.05 was considered significant. 


\section{Results}

Two hundred participants answered the survey. Of these, 134 (67\%) work in academic centers, $58(20 \%)$ in community hospitals, and six $(3 \%)$ in private clinics. One hundred twenty-nine (64\%) were consultants, 42 (21\%) were attending surgeons, and 29 (14\%) were residents. Sixty-two (31\%) surgeons have a work experience of 11-20 years, 55 (27\%) of 5-10 years, 39 (19\%) of less than 5 years, $23(11 \%)$ more than 30 years, and 21(10\%) of 21-30 years. Only 32 were females (16\%). One hundred seventy-two (86\%) surgeons were confident that AI will improve their acute care surgery practice, 17 (8.5\%) thought that it will not affect their job, while 6 thought that it will make it more difficult (3\%).

Seventy-five surgeons (37.5\%) perform minimally invasive surgery in $51-75 \%$ of their procedures, $44(22 \%)$ in $25-50 \%$ of their procedures, $38(19 \%)$ in $76-90 \%$ of their procedures, $24(12 \%)$ in less than $25 \%$ of their procedures, $12(6 \%)(12 / 200)$ in more than $90 \%$ of their procedures while seven surgeons (3.5\%) do not perform minimally invasive surgery. Minimally invasive surgery was performed in both elective and emergency surgery by 149 surgeons (74.5\%), only in elective surgery by 39 (19\%) surgeons, and only in emergency surgery by 8 surgeons (4\%). Fifty surgeons (25\%) were trained, robotic surgeons. Only 19 (9.5\%) were currently performing robotic surgery.

One hundred twenty-six (63\%) surgeons do not have a robotic system in their institution, and for those who have it, it was mainly used for elective surgery. One hundred ten surgeons (55\%) have experience in the 3D system of vision which was mainly in elective surgery. Only 100 surgeons (50\%) were able to define different AI terminologies like general and narrow AI, machine and deep learning, supervised and unsupervised learning, computer vision and natural language processing. Seventy-seven surgeons (38.5\%) read AI-based surgical articles and feel comfortable with their details, whereas $56(28 \%)$ didn't read articles about AI (Table 1, Additional file 2: Appendix 2). Seventy-seven (38.5\%) surgeons think that AI can extremely improve emergency and trauma surgery, and 99 (49.5\%) were highly interested in courses or research projects about the application of AI in emergency surgery (Fig. 1a, b). The majority of the participants thought that they are quick adopters for new technologies Fig. 1c.

The majority of surgeons believe that AI in emergency surgery can be useful to support peri-operative decision making, improved surgical vision, surgical practice, training, and education (Table 1$)$. The highest areas were training and education (61.5\%), perioperative decision making (59.5\%), and improved surgical vision (53\%), (Table 1). 93\% of the surgeons thought that high technologies such as the da Vinci System, I-Drive, and Ligasure should be included in future research. The majority (93\%) of the surgeons want to be involved in future research. Additional file 3: Appendix 3 summarises the suggested research topics by the participants.

Seventy-nine surgeons (39.5\%) systematically collect clinical data in their practice (videos, images, and databases), 61 (30.5\%) occasionally collect data (videos of surgical procedures), and 39 (19.5\%) collected data by official requests. The majority of surgeons $(149,74.5 \%)$ were confident that AI will be available in their setting in the future.

There was no statistically significant difference between males and females in ability, interest in training and expectations of AI ( $p$ values $0.91,0.82$, and 0.28 , respectively, Mann-Whitney U test).

There was also no statistically significant difference between residents, attending surgeons and consultants in ability, interest in training and expectations of AI ( $p$ values $0.82,0.82$, and 0.93 , respectively, Kruskal-Wallis test). Ability was significantly correlated with interest and expectations $(p<0.0001$ Pearson rank correlation, rho 0.42 and 0.47 , respectively) but not with experience $(p=0.9$, rho -0.01$)$ (Table 2).

\section{Discussion}

Our study has shown that the majority of our participants perform minimally invasive surgery in emergency surgery. Nevertheless, only $25 \%$ are trained to perform robotic surgery and less than $10 \%$ currently practice it which highlights the need for more training in this area. Furthermore, more than $60 \%$ of the participants do not have a robotic system in their hospitals. Females have shown similar interest in AI in emergency and trauma surgery to males.

The application of innovative technologies can improve decision making and surgical outcomes. This usually follows an S-shaped curve having 3 phases: (1) the introduction phase, (2) the performance improvement phase, and finally (3) the plateau phase. This is followed by augmentation of its use by replacement of existing standards [3, $12,13]$. Robotic surgery is an evolution of laparoscopic surgery which has a high magnification of the 3-dimensional views, reduction or elimination of hand tremors, instruments that articulate to extreme angles, and comfortable ergonomics and platforms [14]. Several studies have shown that robotic surgery is safe and feasible in the emergency setting. The main obstacle to its adoption is the lack of training and accessibility [15-17]. The current study clearly shows that trauma and acute care surgeons are enthusiasts and supportive of the use of AI in their clinical practice. 


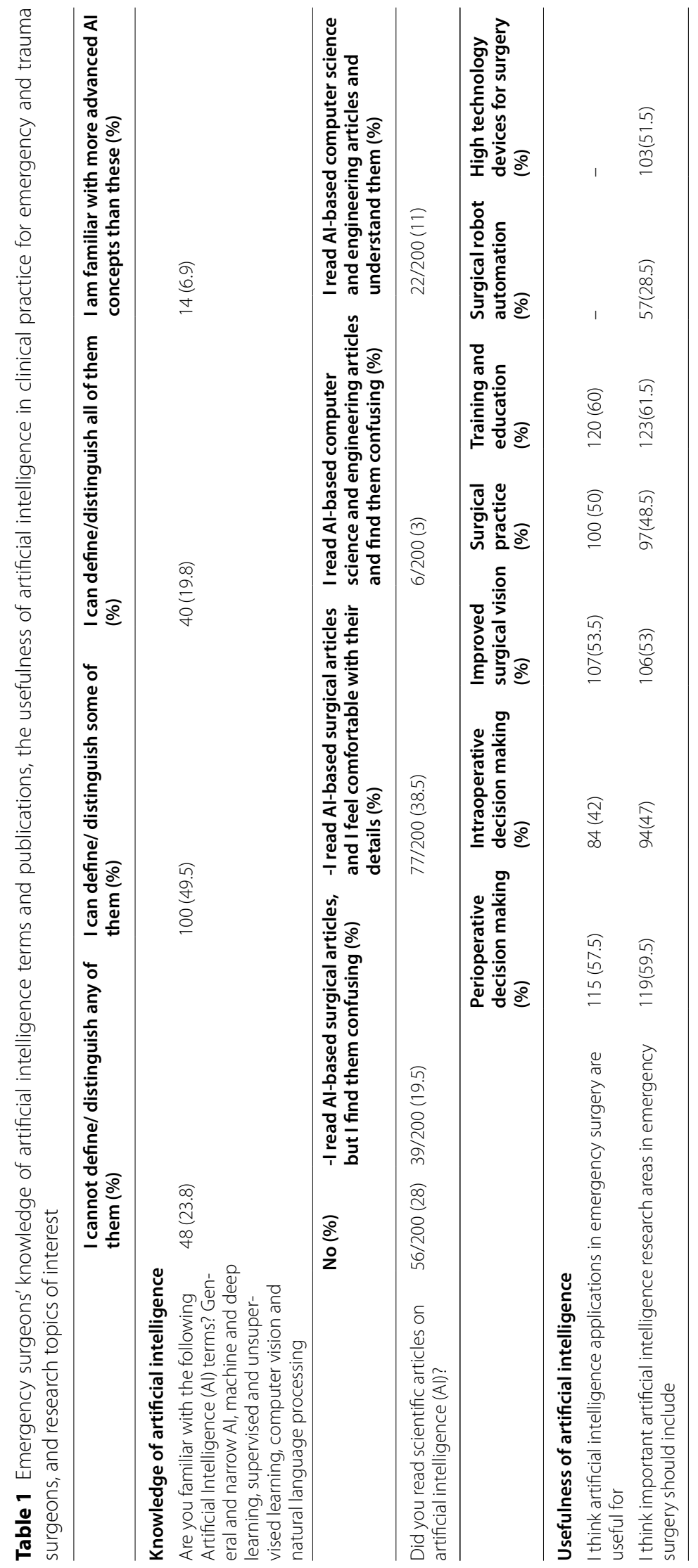




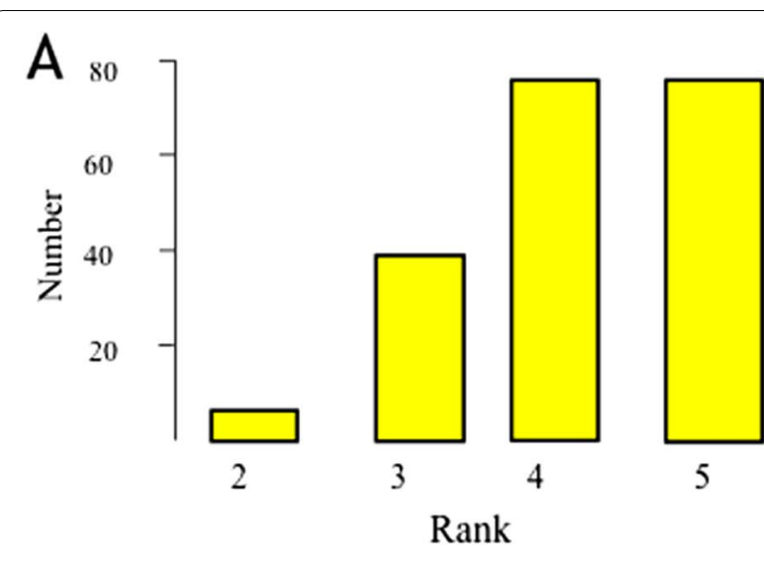

The participants supported research in autonomous actions in surgery [18]. Intelligent technologies may improve vision by using 3D systems, "feel" the thickness of tissues to cut them (using surgical staples) or create vessel fusion (by adjusting energy delivery to the tissue). These actions are achieved by using mathematical algorithms which determine the right action, at the right time, with minimal human supervision.

The majority of participants thought that AI tools are useful in supporting decision making, which may improve clinical outcomes. Critical decision making in the acute care setting is a complex individual clinical reasoning cognitive process affected by emotions, limited time, lack of information, and high risk. Loftus et al. [19] showed that traditional clinical-decision support sys-

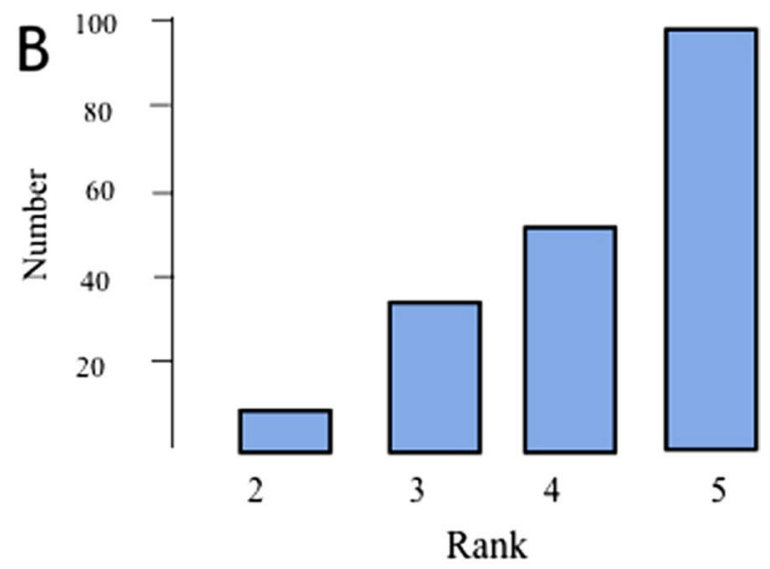
tems are time-consuming because of manual data management and do not consider the nonlinear relationship among multiple non-static variables, decreasing accuracy. AI models, fed with live streaming intraoperative and electronic health record data, integrated with bedside assessment and human intuition could improve critical decision-making.

For example, in performing a safe laparoscopic cholecystectomy, the fulfilment of the criteria of critical view safety (CVS) are required to prevent bile duct injuries, despite that a bile duct injury can occur in managing severe cholecystitis. Deep learning models, built using high quality video-reporting datasets, could assist emergency surgeons in intra-operative decision making, in performing a safe laparoscopic cholecystectomy [20]. In practice, it means that CVS is assessed using computer vision, and the anatomy will be segmented on the operating room screen in safe and dangerous (NO-GO) zones of dissection, liver, gallbladder, and hepatocystic triangle during laparoscopic cholecystectomy, to decrease intraoperative errors in visual perception and judgment leading to misinterpretation of anatomy.[21].

The implementation of decision support systems based on Artificial Neural Networks is limited by: (1) the quality and reliability of medical information, (2) the lack of transparency in the decision-making process, (3) the selection and development of personnel capable of effectively using and maintaining intelligent systems, (4)the high cost and (5) and finally security issues [22].

Large international secured databases may facilitate the development of highly reliable and accurate AI algorithms. It is important to acknowledge that the WSES was very supportive of this issue by developing surgical registers to collect large-high-quality international data [23]. Participating in these international databases with high-quality data will improve the development of useful AI tools. Nevertheless, errors may occur when developing AI algorithms. Accountability for these 
Table 2 Correlations between surgical experience, ability, interest, and expectations of artificial intelligence; Rho and $p$ value of Spearman rank correlation

\begin{tabular}{|c|c|c|c|c|c|}
\hline & Statistics & Surgical experience & Ability & Interest & Expectations \\
\hline \multirow[t]{2}{*}{ Surgical experience } & Rho & - & -0.01 & -0.11 & -0.06 \\
\hline & $p$ value & - & 0.9 & 0.11 & 0.42 \\
\hline \multirow[t]{2}{*}{ Ability } & Rho & -0.01 & - & 0.42 & 0.47 \\
\hline & $p$ value & 0.9 & - & $<0.0001$ & $<0.0001$ \\
\hline \multirow[t]{2}{*}{ Interest } & Rho & -0.11 & 0.42 & - & 0.66 \\
\hline & $p$ value & 0.11 & $<0.0001$ & - & $<0.0001$ \\
\hline \multirow[t]{2}{*}{ Expectations } & Rho & -0.06 & 0.47 & 0.66 & - \\
\hline & $p$ value & 0.42 & $<0.0001$ & $<0.0001$ & - \\
\hline
\end{tabular}

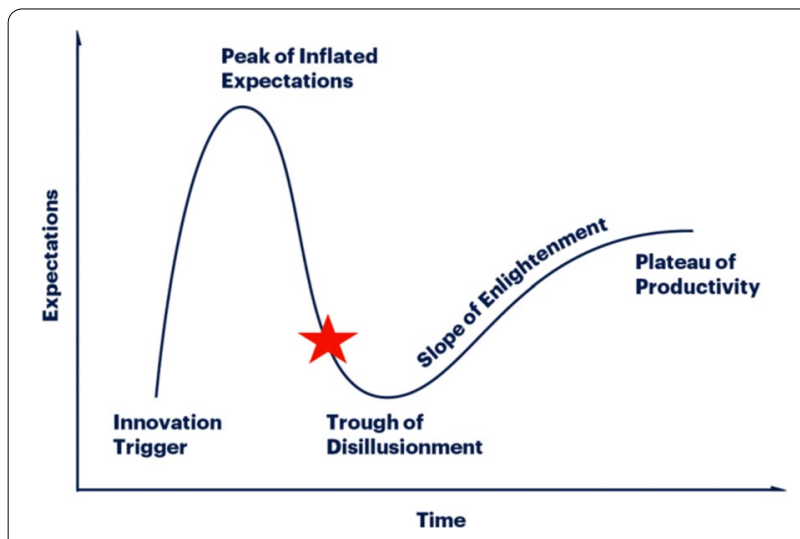

Fig. 2 The Gartner Hype Cycle methodology describes how the perceived value of a given technology evolves

errors poses an important ethical dilemma. O'Sullivan et al. [24] classified responsibility in autonomous robotic surgical procedures into accountability, liability, and culpability. Supervision of the treating surgeon during the early phase of AI implementation is highly important. Other legal issues include privacy, cybercrime, following ethical standards, and human representation [25].

The Gartner Hype Cycle methodology [26] describes how the perceived value of a given new technology evolves in several phases (Fig. 2): the initial enthusiasm phase, the mass adoption and evaluation phase, and finally the maturity phase after technology improvement. The limited access of trauma and emergency surgeons to robotic surgery due to unavailability, high cost, and lack of training have delayed its implementation in clinical practice. We think that AI applications will improve emergency surgery outcomes. The current survey shows that the ability in adopting new technologies is significantly correlated with interest and expectations.

\section{Limitations}

This study has certain limitations. First, the response rate is low, and the sample size is relatively small. This increase the risk of selection bias. Those who are interested in AI may have responded with a relatively higher percentage reported in those being trained in AI. This may not reflect reality. Second, this study is survey-based which depends on subjective opinions and carries the risk of recall bias. Third, we depended on surface validity and expert opinion in developing this questionnaire. Nevertheless, we think that the information we gained is useful and helps guide us in exploring this important area.

\section{Conclusions}

The ARIES survey showed that the implementation of AI in the emergency and trauma setting is still in an early phase, despite the high interest showed by emergency surgeons invited to join the ARIES project. Its progression needs focusing research in the most useful fields of interest to improve patients' outcomes. Emergency and trauma surgeons claimed to be involved. The support of emergency and trauma surgeons by health care systems and industries is essential for the progress of $\mathrm{AI}$ in their setting which will be augmented by proper research and training programs in this area.

\section{Abbreviations}

Al: Artificial Intelligence; WSES: World Society of Emergency Surgery; ARIES: Artificial intelligence in emergency and trauma surgery; ES: Emergency surgery.

\section{Supplementary Information}

The online version contains supplementary material available at https://doi. org/10.1186/s13017-022-00413-3.

Additional file 1. Appendix 1: ARIES survey form.

Additional file 2. Appendix 2: Some Artificial Intelligence definitions [2-8-14]. 
Additional file 3. Appendix 3: Research topics suggested from ARIES participants.

Additional file 4. Appendix 4: ARIES collaborative group.

\section{Acknowledgements}

Many thanks to the ARIES collaborative group for participation (e-mail addresses are listed in the Additional file 4: Appendix 4)

\section{Authors' contributions}

BDS conceived the study and wrote the questionnaire; the experts' panel (AG, $T C, M P, P M, L M, A L i, W B, E C, F A Z$ ) revised the questionnaire, and the ARIES project was submitted to the evaluation of the WSES steering committee (FC, FCO, MS, SDS, LA, MS, EEM, YK, AL) for approval and diffusion. BDS collected data and wrote the manuscript; FAZ carried out the statistical analysis; FAZ, FC, $A G$, and $E C$ read and revised the first draft of the paper. All the authors read and approved the final manuscript.

\section{Funding}

No funding received for this article.

Availability of data and materials

Not applicable.

\section{Declarations}

Ethics approval and consent to participate

Not applicable.

\section{Consent for publication}

Not applicable.

\section{Competing interests}

The authors declare that they have no competing interests.

\section{Author details}

'Department of Emergency and Metabolic Minimally Invasive Surgery, Centre Hospitalier Intercommunal de Poissy/Saint Germain en Laye, 10 Rue de Champ Gaillard, Poissy Cedex, France. ${ }^{2}$ Department of Surgery, College of Medicine and Health Sciences, UAE University, Al-Ain, United Arab Emirates. ${ }^{3}$ Department of General Surgery, Ospedale Civile "Madonna del Soccorso", San Benedetto del Tronto, AP, Italy. ${ }^{4}$ Department of General Surgery, Macerata Hospital, Macerata, Italy. ${ }^{5}$ Department of Surgery, Pisa University Hospital, Pisa, Italy. ${ }^{6}$ Department of General Surgery, University Hospital of Pavia, Pavia, Italy. ${ }^{7}$ RCAD, Strasbourg, France. ${ }^{8}$ Department of Emergency and Trauma Surgery, Rambam Health Campus, Haifa, Israel. ${ }^{9}$ Department of Surgery, School of Medicine and the Ernest E. Moore Shock Trauma Center at Denver Health, University of Colorado, Denver, CO, USA. ${ }^{10}$ Abdominal Center, Helsinki University Hospital and University of Helsinki, Helsinki, Finland. ${ }^{11}$ Department of Surgical Disciplines, Immanuel Kant Baltic Federal University, Regional Clinical Hospital, Kaliningrad, Russia. ${ }^{12}$ Fondazione Policlinico Universitario A. Gemelli IRCCS, Rome, Italy. ${ }^{13}$ Department of General and Robotic Surgery, The Brooklyn Hospital Center, New York, USA. ${ }^{14}$ Division of General, Emergency Surgery and New Technologies, Ospedale Civile Di Baggiovara, Azienda Ospedaliero - Universitaria Di Modena, Modena, Italy. ${ }^{15}$ Hepato-Bilio-Pancreatic Minimally Invasive Surgery, Poliambulanza Foundation Hospital, Brescia, Italy. ${ }^{16}$ Department of Surgery, Letterkenny University Hospital Ireland, Letterkenny, Ireland. ${ }^{17}$ Department of Trauma and Acute Care Surgery, Scripps Memorial Hospital, La Jolla, CA, USA. ${ }^{18}$ Department of Emergency and Trauma Surgery, Bufalini Hospital, Cesena, Italy.

Received: 27 October 2021 Accepted: 24 January 2022

Published online: 10 February 2022

\section{References}

1. Bellman R. An introduction to artificial intelligence: can computers think? San Francisco: Boyd \& Fraser Pub Co; 1978.
2. Hashimoto DA, Rosman G, Rus D, Meireles OR. Artificial intelligence in surgery: promises and perils. Ann Surg. 2018;268(1):70-6. https://doi.org/ 10.1097/SLA.0000000000002693.PMID:29389679:PMCID:PMC5995666.

3. Vitiello V, Lee SL, Cundy TP, Yang GZ. Emerging robotic platforms for minimally invasive surgery. IEEE Rev Biomed Eng. 2013;6:111-26. https://doi. org/10.1109/RBME.2012.2236311 (Epub 2012 Dec 24 PMID: 23288354).

4. Mascagni P, Longo F, Barberio M, Seeliger B, Agnus V, Saccomandi $P$, Hostettler A, Marescaux J, Diana M. New intraoperative imaging technologies: Innovating the surgeon's eye toward surgical precision. J Surg Oncol. 2018;118(2):265-82.

5. Mascagni P, Vardazaryan A, Alapatt D, Urade T, Emre T, Fiorillo C, Pessaux P, Mutter D, Marescaux J, Costamagna G, Dallemagne B. Artificial intelligence for surgical safety: automatic assessment of the critical view of safety in laparoscopic cholecystectomy using deep learning. Ann Surg. 2021 May 24.

6. Madani A, Namazi B, Altieri MS, Hashimoto DA, Rivera AM, Pucher PH, Navarrete-Welton A, Sankaranarayanan G, Brunt LM, Okrainec A, Alseidi A. Artificial intelligence for intraoperative guidance: using semantic segmentation to identify surgical anatomy during laparoscopic cholecystectomy. Ann Surg. 2021 May 24.

7. Mascagni P, Padoy N. OR black box and surgical control tower: recording and streaming data and analytics to improve surgical care. J Visceral Surg. 2021 Mar 9.

8. Eysenbach G. Improving the quality of Web surveys: the checklist for reporting results of internet E-surveys (CHERRIES). J Med Internet Res. 2004;6(3): e34.

9. Jones TL, Baxter MA, Khanduja V. A quick guide to survey research. Ann R Coll Surg Engl. 2013;95(1):5-7. https://doi.org/10.1308/003588413X13511 609956372.PMID:23317709;PMCID:PMC3964639.

10. Ball HL. Conducting online surveys. J Hum Lact. 2019;35(3):413-7. https://doi.org/10.1177/0890334419848734 (Epub 2019 May 14 PMID: 31084575).

11. Eysenbach G, Wyatt J. Using the Internet for surveys and health research. J Med Internet Res. 2002;4(2):E13. https://doi.org/10.2196/jmir.4.2.e13.

12. Loftus TJ, Filiberto AC, Balch J, Ayzengart AL, Tighe PJ, Rashidi P, Bihorac A, Upchurch GR Jr. Intelligent, autonomous machines in surgery. J Surg Res. 2020;253:92-9. https://doi.org/10.1016/j.jss.2020.03.046.

13. Gumbs AA, Perretta $S$, d'Allemagne B, Chouillard E. What is artificial intelligence surgery? Art Int Surg 2021;1:1-10. https://doi.org/10.20517/ais. 2021.01

14. Gumbs AA, De Simone B, Chouillard E. Searching for a better definition of robotic surgery: is it really different from laparoscopy? Mini-invasive Surg 2020;4:90. https://doi.org/10.20517/2574-1225.2020.110

15. Gumbs AA, Frigerio I, Spolverato G, Croner R, Illanes A, Chouillard E, Elyan E. Artificial intelligence surgery: how do we get to autonomous actions in surgery? Sensors. 2021;21:5526. https://doi.org/10.3390/s21165526.

16. Cubas R, Garcia M, Mukherjee K. Robotic repair of incarcerated morgagni hernia in an adult on the acute care surgery service. Rev Fac Cien Med Univ Nac Cordoba. 2021;78(1):91-4. https://doi.org/10.31053/1853.0605. V78.n1.28299 (PMID: 33787029).

17. Kudsi OY, Bou-Ayash N, Chang K, Gokcal F. Perioperative and midterm outcomes of emergent robotic repair of incarcerated ventral and incisional hernia. J Robot Surg. 2021;15(3):473-81. https://doi.org/10.1007/ S1 1701-020-01130-2 (Epub 2020 Jul 28 PMID: 32725328).

18. Ceccarelli G, Pasculli A, Bugiantella W, De Rosa M, Catena F, Rondelli F, Costa G, Rocca A, Longaroni M, Testini M. Minimally invasive laparoscopic and robot-assisted emergency treatment of strangulated giant hiatal hernias: report of five cases and literature review. World J Emerg Surg. 2020;15(1):37. https://doi.org/10.1186/s13017-020-00316-1.PMID:32487 136:PMCID:PMC7268602.

19. Loftus TJ, Tighe PJ, Filiberto AC, Efron PA, Brakenridge SC, Mohr AM, Rashidi P, Upchurch GR Jr, Bihorac A. Artificial intelligence and surgical decision-making. JAMA Surg. 2020;155(2):148-58. https://doi.org/10. 1001/jamasurg.2019.4917.PMID:31825465:PMCID:PMC7286802.

20. Mascagni P, Vardazaryan A, Alapatt D, Urade T, Emre T, Fiorillo C, Pessaux P, Mutter D, Marescaux J, Costamagna G, Dallemagne B, Padoy N. Artificial Intelligence for surgical safety: automatic assessment of the critical view of safety in laparoscopic cholecystectomy using deep learning. Ann Surg. 2020. https://doi.org/10.1097/SLA.0000000000004351.

21. Madani A, Namazi B, Altieri MS, Hashimoto DA, Rivera AM, Pucher PH, Navarrete-Welton A, Sankaranarayanan G, Brunt LM, Okrainec A, Alseidi 
A. Artificial intelligence for intraoperative guidance: using semantic segmentation to identify surgical anatomy during laparoscopic cholecystectomy. Ann Surg. 2020. https://doi.org/10.1097/SLA.0000000000004594.

22. Litvin A, Korenev S, Rumovskaya S, et al. WSES project on decision support systems based on artificial neural networks in emergency surgery. World J Emerg Surg. 2021;16:50. https://doi.org/10.1186/ s13017-021-00394-9.

23. World Society of Emergency Surgery, Clinical Registers. Available at https://www.clinicalregisters.org Accessed on October 13, 2021

24. O'Sullivan S, Nevejans N, Allen C, Blyth A, Leonard S, Pagallo U, Holzinger K, Holzinger A, Sajid MI, Ashrafian H. Legal, regulatory, and ethical frameworks for development of standards in artificial intelligence (Al) and autonomous robotic surgery. Int J Med Robot. 2019;15(1): e1968. https:// doi.org/10.1002/rcs.1968 (PMID: 30397993).

25. Zhou XY, Guo Y, Shen M, Yang GZ. Application of artificial intelligence in surgery. Front Med. 2020;14(4):417-30. https://doi.org/10.1007/s11684020-0770-0 (Epub 2020 Jul 23 PMID: 327054).

26. Gartner. Gartner Hype Cycle. Available at https://www.gartner.com/en/ research/methodologies/gartner-hype-cycle Accessed October 13, 2021.

\section{Publisher's Note}

Springer Nature remains neutral with regard to jurisdictional claims in pub-

lished maps and institutional affiliations.

- fast, convenient online submission

- thorough peer review by experienced researchers in your field

- rapid publication on acceptance

- support for research data, including large and complex data types

- gold Open Access which fosters wider collaboration and increased citations

- maximum visibility for your research: over 100M website views per year

At BMC, research is always in progress.

Learn more biomedcentral.com/submissions 